\title{
FAIR INSURANCE PREMIUM RATE IN CONNECTED SEIR MODEL UNDER EPIDEMIC OUTBREAK*
}

\author{
Alexey A. Chernov ${ }^{1, * *}$, Aleksandr A. Shemendyuk ${ }^{2}$ \\ AND MARK Y. KELBERT ${ }^{1}$
}

\begin{abstract}
In this paper, we aim to determine an optimal insurance premium rate for health-care in deterministic and stochastic SEIR models. The studied models consider two standard SEIR centres characterised by migration fluxes and vaccination of population. The premium is calculated using the basic equivalence principle. Even in this simple set-up, there are non-intuitive results that illustrate how the premium depends on migration rates, the severity of a disease and the initial distribution of healthy and infected individuals through the centres. We investigate how the vaccination program affects the insurance costs by comparing the savings in benefits with the expenses for vaccination. We compare the results of deterministic and stochastic models.
\end{abstract}

Mathematics Subject Classification. 92D30, 37N25, 91G60, 34A30.

Received October 26, 2020. Accepted May 17, 2021.

\section{INTRODUCTION}

Epidemics cause severe damage to social welfare and can result in a massive loss of working days. Many models have been developed to estimate the dynamics of epidemics, e.g. pioneer studies $[6,19,25,32]$ and later works $[3,7,8,16,18,21]$. One of the most fundamental works with strict mathematical and medical approaches is [10]. Investigation of these models can help understanding the key points of the phenomena and determine the optimal vaccination strategy, which could stop the spread of the pandemic and reduce the economic costs.

Vaccination is in a focus of intensive research nowadays. The question of optimal vaccine allocation strategies occupies a significant niche in epidemiology. Urgent decisions are required when an epidemic starts to spread. The vaccination strategies and effects of different vaccination methods were studied since 1970s, including $[18,24]$ and more recent $[2,4,8,17,21,27-29]$. Optimal vaccine allocation for limited stock was investigated in deterministic [12] and in stochastic [37] cases. The problem of optimal vaccine allocation in two interacting centres with limited vaccine stock was studied in deterministic case in [9].

Along with this, the health-care premium is directly influenced by the health status of the population. The population bares the epidemic costs either through the tax system or directly, buying a medical insurance. For

${ }^{*}$ The authors thank an anonymous reviewer for the helpful comments and suggestions. This paper is prepared within the framework of the Russian Academic Excellence Project "5-100".

Keywords and phrases: Optimal premium level, SEIR, epidemics, vaccination.

${ }^{1}$ National Research University Higher School of Economics, Laboratory of Stochastic Analysis and its Applications, Moscow, Russia.

${ }^{2}$ University of Lausanne, Department of Actuarial Science, Lausanne, Switzerland.

** Corresponding author: chernov1251@gmail.com

(C) The authors. Published by EDP Sciences, 2021 
definiteness, we consider here an insurance scheme though the calculations would not change significantly if the expenses are paid from a centralized health-care system. Assume that after the start of epidemic a manager of health-care office may either order a vaccine for a particular individual, or issue a mandatory extension to the current health-care insurance policy that covers future expenses in the case this individual will need a treatment. There are numerous principles that determine the health-care premium (e.g., Esscher, exponential, variance principles, see [30]), which imply the positive safety loading. In our work, we use the basic equivalence principle

$$
\mathbb{E}[\text { benefit outgo }]=\mathbb{E}[\text { premium income }] \text {. }
$$

to highlight the non-profitability of a public health-care office, which is highly expected during the outbreak.

The aim of this work is to investigate the optimal health-care premium rate $\pi$ in deterministic and stochastic connected SEIR centres. We consider different scenarios (different centres' characteristics), investigate the dependence of premium $\pi$ on the amount of vaccine available, infection rates, migration intensities, sizes of population and vaccine allocation strategies. Further, we compare the results of different scenarios for stochastic and deterministic models. We consider parameters that are relevant to the recent COVID-19 epidemic with mild contamination scenario with reproduction number $\mathcal{R}_{0}=2$ (see, e.g., [14]), and with $\mathcal{R}_{0}=4$ for more aggressive virus stamps (see, e.g., [23]).

To do so, we consider deterministic and stochastic SEIR models with two connected centres and constant migration fluxes. The models consider four compartments (population groups) at time $t$ : susceptible to a disease $S(t)$, exposed $E(t)$, infected $I(t)$ and removed $R(t)$ persons. The last compartment consists of subjects who either acquired an immunity after suffering from the disease and will not become ill again, or succumbed to the disease. The centres could represent cities, countries, health-care facilities, etc. We calculate the health-care premium rate for different parameters of the model by optimally (in some sense) allocating the vaccine among the centres.

Epidemic models have been proposed in different actuarial frameworks in [13, 22]. The paper [13] considers constant infection and recovery rates in an isolated SIR model without migration. The authors present calculations of annuity premiums and annuity benefits, lump-sum benefits and death benefits. They also propose power series solutions for evaluating the dynamic of the model. Then the premium adjustments are introduced and the method is applied to real data sets: great plague in Eyam in 1665 and SARS epidemic in Hong Kong in 2003. In [22], the authors consider a time-continuous Markov model of an epidemic spread with one centre and different epidemic scenarios: general and fatal epidemics, a case with exponentially-dependent rates, and a single-time change of the infection rate model.

The paper is organised as follows. In Section 2, we introduce a deterministic SEIR model, basic notions and two measures of optimality. In Section 3, a Markov chain model is developed that allows to account for the extinction of epidemics. In Section 4, the simulation results are presented. In Section 5, we draw conclusions and outline the possible extensions of the current research. Finally, in Appendix A we calculate the reproduction number for two connected SEIR centres.

\section{Deterministic MODEL}

\subsection{Connected SEIR model}

The classical SEIR model for $n$ centres with migration fluxes is governed by the following system of ODEs:

$$
\begin{aligned}
\frac{\mathrm{d} S_{i}}{\mathrm{~d} t} & =-\frac{\beta_{i}}{N_{i}} S_{i} I_{i}-\sum_{j \neq i} k_{i j} S_{i}+\sum_{j \neq i} k_{j i} S_{j} \\
\frac{\mathrm{d} E_{i}}{\mathrm{~d} t} & =\frac{\beta_{i}}{N_{i}} S_{i} I_{i}-\sum_{j \neq i} k_{i j} E_{i}+\sum_{j \neq i} k_{j i} E_{j}-\sigma E_{i} \\
\frac{\mathrm{d} I_{i}}{\mathrm{~d} t} & =\sigma E_{i}-\gamma_{i} I_{i}-\sum_{j \neq i} l_{i j} I_{i}+\sum_{j \neq i} l_{j i} I_{j} \\
\frac{\mathrm{d} R_{i}}{\mathrm{~d} t} & =\gamma_{i} I_{i}-\sum_{j \neq i} k_{i j} R_{i}+\sum_{j \neq i} k_{j i} R_{j} \\
S_{i}(0) & =S_{i, 0}, E_{i}(0)=E_{i, 0}, I_{i}(0)=I_{i, 0}, R_{i}(0)=0,
\end{aligned}
$$


where $i=1,2, \ldots, n ; \beta_{i}$ is the infection rate, $\sigma$ is the rate of transfer from exposed to infective and $\gamma_{i}$ is recovery rate; $k_{i j}$ are the migration rates of susceptible, exposed and removed and $l_{i j}$ are the migration rates of infected groups from $i$-th to $j$-th centre; $S_{i, 0}, E_{i, 0}, I_{i, 0}$ and $R_{i, 0}$ are initial numbers of susceptible, exposed, infected and removed persons in $i$-th centre. The population in $i$-th centre is then $N_{i}(t)=S_{i}(t)+E_{i}(t)+I_{i}(t)+R_{i}(t)$. The total population is constant $N \equiv N(t)=\sum_{i=1}^{n} N_{i}(t)$ in view of (2.1). The parameter $T_{e}=\frac{1}{\gamma}$ stands for the mean duration of the disease, $T_{l}=1 / \sigma$ is the mean duration of the latent period. The main parameter of an epidemiological model is the so-called reproduction number $\mathcal{R}_{0}[1,15,20,31,32,35]$. The epidemiological definition of $\mathcal{R}_{0}$ is the average number of secondary cases produced by one infected individual introduced into a population of susceptibles where an infected individual has acquired the disease, and susceptible individuals are healthy but can acquire the disease. Note that $\mathcal{R}_{0}$ characterizes the severity of epidemic outbreak. For the system (2.1) $\mathcal{R}_{0}$ is computed in Appendix A by the next generation method, see [11, 26, 35]. A similar analysis in the case of SIR model with migration fluxes is given in [34].

The key object of the model is the time moment $T$ when the epidemic stops. It is widely known that nontrivial solutions of (2.1) have exponential character. Therefore, we can never obtain pure zero number of infectives by integrating the system. It is natural to say that if there are less infectives than a certain value, then the epidemic is assumed to be suppressed. We therefore set the threshold percentage $\theta$ of living population, which is sufficient to assume that the epidemic is suspended:

$$
T=\inf \left\{t: \sum_{i=1}^{n}\left[I_{i}(t)+E_{i}(t)\right]<\theta \cdot N\right\} .
$$

\subsection{Infectivity and susceptibility times}

One of the key functionals is

$$
A_{i}(T)=\int_{0}^{T} I_{i}(t) \mathrm{d} t, \quad i=1, \ldots, n
$$

which describes the total number of lost working days during the epidemic in $i$-th centre. This functional also allows us to measure the medical expenses for treating infected persons.

Since we are interested in the total number of lost working days in the network, we sum over $i$ and define

$$
A_{T} \equiv \sum_{i=1}^{n} A_{i}(T)=\sum_{i=1}^{n} \int_{0}^{T} I_{i}(t) \mathrm{d} t .
$$

In order to evaluate the treatment costs for patients $I$, we introduce a discount factor into the functional $A$, taking into account a daily discount rate $\delta$. The formula (2.3) takes the form:

$$
A_{T}^{\delta}=\sum_{i=1}^{n} \int_{0}^{T} \exp (-\delta t) I_{i}(t) \mathrm{d} t
$$

During the epidemic a health-care office collects premiums from healthy and exposed individuals with some rate (will be introduced in Sect. 2.3). Total exposure to premiums of all susceptible and exposed persons in $i$-th centre is

$$
B_{i}^{\delta}(T)=\int_{0}^{T} \exp (-\delta t)\left[S_{i}(t)+E_{i}(t)\right] \mathrm{d} t, \quad i=1, \ldots, n
$$


By summing over $i$, we define

$$
B_{T}^{\delta} \equiv \sum_{i=1}^{n} B_{i}^{\delta}(T)=\sum_{i=1}^{n} \int_{0}^{T} \exp (-\delta t)\left[S_{i}(t)+E_{i}(t)\right] \mathrm{d} t .
$$

\subsection{Premium calculation}

The premium is calculated according to the standard equivalence principle (1.1). On the liability side, the benefits are paid by the health-care office to infected persons with constant rate $c_{1}$. The office pays a lump sum $c_{2}$ to every person transferred to the removed group (i.e. got the immunity). In line with [22], we assume that all claims are settled at time $T$ when the epidemic is extinct. Finally, the health-care office purchases/invests in vaccine supplies $\nu$, each unit having a cost of $c_{3}$. Then, the expected liability is

$$
\mathbb{E}[\text { benefit outgo }]=c_{1} A_{T}^{\delta}+c_{2} \exp (-\delta T) \sum_{i=1}^{n} R_{i}(T)+c_{3} \nu
$$

Note that in the deterministic model the outgoing benefits coincide with its expected value.

On the other hand, the health-care office collects constant premiums $\pi$ from the susceptible and exposed population until they become ill or the epidemic has ended. Thus, the expected income of the health-care office is

$$
\mathbb{E}[\text { income }]=\pi B_{T}^{\delta}
$$

Note that this premium collection mechanism is fair to the population, since it only charges the susceptible and exposed population. In real life, the insurance premiums would be paid by everyone during the whole epidemic period.

By equivalence principle (1.1) and expressions (2.6) and (2.7), we get

$$
\pi=\frac{1}{B_{T}^{\delta}}\left(c_{1} A_{T}^{\delta}+c_{2} \exp (-\delta T) \sum_{i=1}^{n} R_{i}(T)+c_{3} \nu\right) .
$$

\subsection{Optimal vaccine allocation}

Once the epidemic starts and the health-care office possesses vaccine amount $\nu$, it is important to wisely allocate the vaccine among the centres. In real life the vaccination is not instantaneous and people prefer to wait for the vaccination, rather than risk their health if the vaccine is just recently developed. Let $w_{i}$ be the shares of vaccine stock $\nu$ allocated in $i$-th centre, $i=1, \ldots, n$. In [9] it is shown that the best vaccination time is $t=0$, since any delay in vaccination lets the infection to spread. However, we consider here a more realistic model of gradual vaccination with the vaccination rate at $i$-th centre being $a_{i}$. Therefore, the SEIR model (2.1) 
with vaccine allocation $\left(w_{1}, \ldots, w_{n}\right): w_{i} \geq 0, w_{1}+\ldots+w_{n}=1$ takes the form

$$
\begin{aligned}
\frac{\mathrm{d} S_{i}}{\mathrm{~d} t} & =-\frac{\beta_{i}}{N_{i}} S_{i} I_{i}-k_{i j} S_{i}+k_{j i} S_{j}-a_{i} \cdot \mathbf{1}\left(V_{i}>0\right) \cdot \mathbf{1}\left(S_{i}>0\right) \\
\frac{\mathrm{d} E_{i}}{\mathrm{~d} t} & =\frac{\beta_{i}}{N_{i}} S_{i} I_{i}-k_{i j} E_{i}+k_{j i} E_{j}-\sigma E_{i} \\
\frac{\mathrm{d} I_{i}}{\mathrm{~d} t} & =\sigma E_{i}-\gamma_{i} I_{i}-l_{i j} I_{i}+l_{j i} I_{j} \\
\frac{\mathrm{d} R_{i}}{\mathrm{~d} t} & =\gamma_{i} I_{i}-k_{i j} R_{i}+k_{j i} R_{j} \\
\frac{\mathrm{d} W_{i}}{\mathrm{~d} t} & =a_{i} \cdot \mathbf{1}\left(V_{i}>0\right) \cdot \mathbf{1}\left(S_{i}>0\right)-k_{i j} W_{i}+k_{j i} W_{j} \\
\frac{\mathrm{d} V_{i}}{\mathrm{~d} t} & =-a_{i} \cdot \mathbf{1}\left(V_{i}>0\right) \cdot \mathbf{1}\left(S_{i}>0\right) \\
S_{i}(0) & =S_{i, 0}, E_{i}(0)=E_{i, 0}, I_{i}(0)=I_{i, 0}, R_{i}(0)=0, W_{i}(0)=0, V_{i}(0)=w_{i} \nu
\end{aligned}
$$

where $\mathbf{1}(\cdot)$ is the indicator function, $V_{i}=\nu \omega_{i}$ is the amount of vaccine still available at the centre $i, W_{i}$ is the number of vaccinated individuals at the centre $i$.

One natural way to optimally allocate the vaccine is to minimise the resulting premium (2.8). Since the health-care is assumed to be mandatory, we would like to reduce the premium paid by the susceptibles and exposed as much as possible. More formally, for given vaccine stock and model parameters, we allocate the vaccine in such a way that the health-care premium rate is minimised:

$$
\pi^{*}=\min _{\substack{\left(w_{1}, \ldots, w_{n}\right): w_{i} \geq 0 \\ w_{1}+\ldots+w_{n}=1}} \frac{1}{B_{T}^{\delta}}\left(c_{1} A_{T}^{\delta}+c_{2} \exp (-\delta T) \sum_{i=1}^{n} R_{i}(T)+c_{3} \nu\right)
$$

Alternatively, we can set as a goal to keep low the treatment costs, i.e. have as less lost working days as possible. Therefore, the optimal vaccine allocation is such that the functional $A_{T}$ is minimised:

$$
A_{T}^{*}=\min _{\substack{\left(w_{1}, \ldots, w_{n}\right): w_{i} \geq 0, w_{1}+\ldots+w_{n}=1}} \sum_{i=1}^{n} \int_{0}^{T} I_{i}(t) \mathrm{d} t
$$

Allocation $\left(w_{1}, \ldots, w_{n}\right)$ affects the current number of susceptible persons, which affects $A_{T}^{\delta}, B_{T}^{\delta}$ and $R_{i}(T)$. Here, we also account for the population of a centre: if an exceeding vaccine share is assigned to the centre (more that its population), the excess should be redirected to the other centres.

It is interesting to see the relation between two "optimality" points of view. Both have sensible reasons of treating epidemic - the first is financially oriented, while the second prevents population from being ill.

\section{Stochastic MODEL}

As in the previous sections, we denote the number of susceptible persons at time $t$ as $S(t)$, infected persons as $I(t)$, exposed persons as $E(t)$ and recovered / removed persons as $R(t)$. Here the main goal is to model the infection spread among population assuming small number of initially infected persons.

If the number of infectives is small, the infection can become naturally suppressed, not causing any epidemic outbreak. A stochastic model is generally devoted to such cases, whereas deterministic differential models describe evolution of epidemic in high populations with large number of infected individuals. The algorithm of merging two approaches is proposed in [33]. 
TABLE 1. Transition rates of time continuous Markov chain in case of one centre.

\begin{tabular}{llll}
\hline Event & & Rate & Condition \\
\hline$S \rightarrow S-1, \quad E \rightarrow E+1$ & $\frac{\beta}{N} S I$ & $S>0$ \\
$E \rightarrow E-1, \quad I \rightarrow I+1$ & $\sigma E$ & $E>0$ \\
$I \rightarrow I-1, \quad R \rightarrow R+1$ & $\gamma I$ & $I>0$ \\
Absorbing state & 0 & $E=0, I=0$ \\
\hline
\end{tabular}

All stochastic models are considered to have a discrete state space in continuous time. Also, models have Markov property, i.e. jumps rates from one state to others do not depend on history. These assumptions are natural because in real epidemics the number of people getting infected only depends on the number of contacts with infected persons. We do not study cases when a disease can infect people from other sources (water, air breeze, unsanitary conditions, intentional release of a disease, etc.). Similarly, recovery rate only depends on the host of the infection.

\subsection{Markov chain for one centre}

In the case of one centre model, the transition rates of standard stochastic model with constant coefficients $\beta, \sigma$ and $\gamma$ are presented in Table 1 .

We denote the time of $j$-th jump (switch of state) as $t_{j}$, and the corresponding waiting time (i.e. time between $j-1$-th and $j$-th jumps) as $s_{j}$.

The final time of epidemic is a random variable that depends on the chain evolution. We define the end of epidemic as

$$
T=\inf \{t: I(t)=0, E(t)=0\} .
$$

Simulation algorithm is presented in Algorithm 1. The general approach is to calculate all transition rates at time $t_{j}$ and simulate the waiting time $s_{j}$ (line 4) as exponential random variable with inverse mean equals to sum of all rates. Then generate a standard uniform random variable $u_{j}$ (line 6 ) to determine which event took place. Calculate the probability of an event as its rate divided by sum of all rates (line 7). Finally, choose a corresponding event (lines 8-12).

\subsection{Markov chain for multiple centres}

Here we consider a stochastic model for epidemic in multiple centres. Denote the number of susceptibles in $i$-th centre at time $t$ as $S_{i}(t)$, exposed as $E_{i}(t)$, infectives as $I_{i}(t)$ and recovered / removed as $R_{i}(t)$ for $i=1, \ldots, n$. Then the transition rates for $i, j=1, \ldots, n$ are presented in Table 2 . The end of epidemic is defined as

$$
T=\inf \left\{t: \sum_{i=1}^{n}\left[E_{i}(t)+I_{i}(t)\right]=0\right\}
$$

i.e. the time when there are no exposed and infectives left.

The simulation algorithm of a single Markov chain for $n$ centres is similar to Algorithm 1. However, here the dimension of state space is $4 n-1$, where we have four numbers for susceptible-exposed-infected-recovered quadruple and $n$ centres with constant population throughout the network. Latter reduces the dimension by one. 


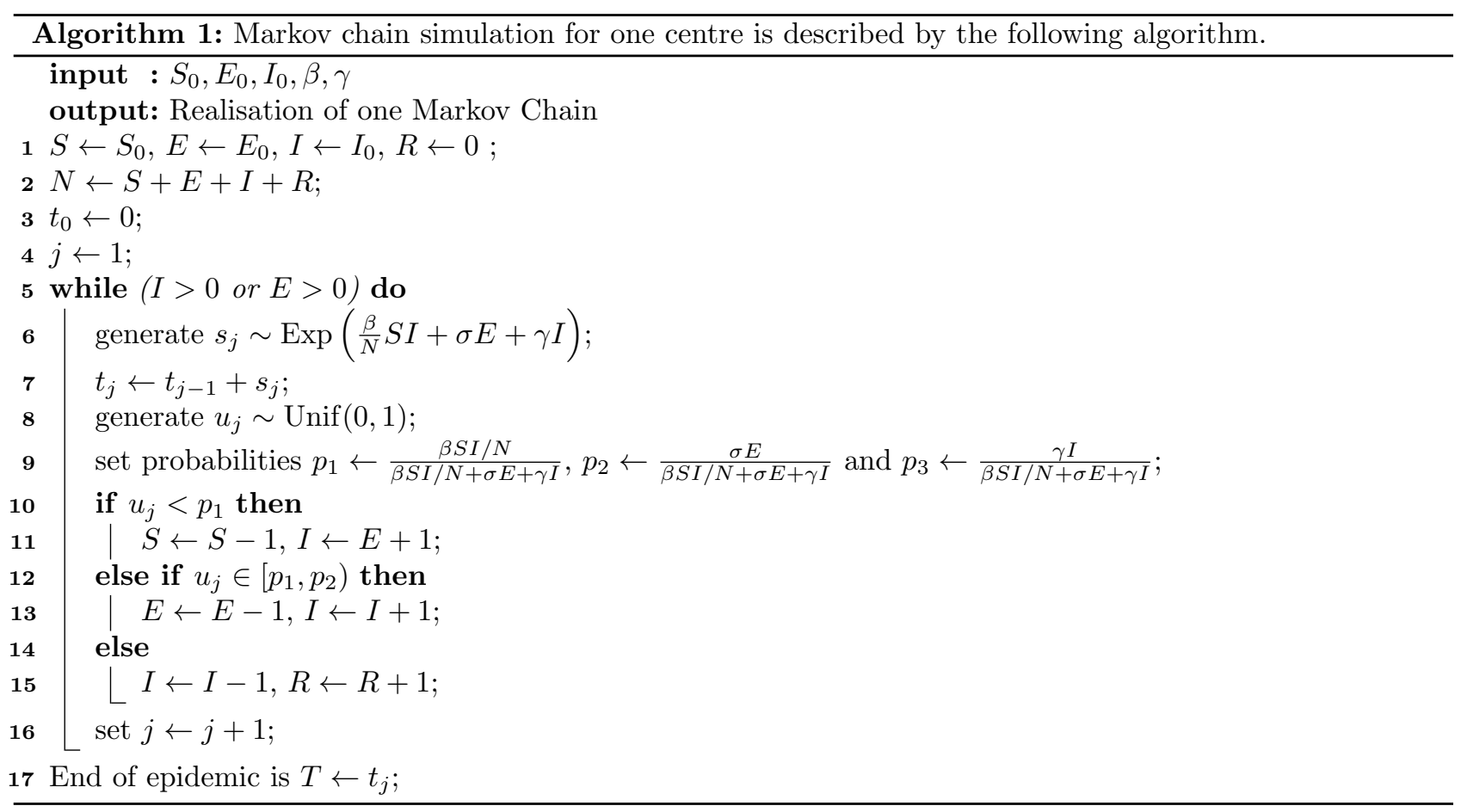

TABLE 2. Transition rates of time continuous Markov chain in case of multiple centres

\begin{tabular}{llll}
\hline Event & & Rate & Condition \\
\hline$S_{i} \rightarrow S_{i}-1, \quad E_{i} \rightarrow E_{i}+1$ & $\beta_{i} S_{i} I_{i} / N$ & $S_{i}>0$ \\
$E_{i} \rightarrow E_{i}-1, \quad I_{i} \rightarrow I_{i}+1$ & $\sigma E_{i}$ & $E_{i}>0$ \\
$I_{i} \rightarrow I_{i}-1, \quad R_{i} \rightarrow R_{i}+1$ & $\gamma_{i} I_{i}$ & $I_{i}>0$ \\
$S_{i} \rightarrow S_{i}-1, \quad S_{j} \rightarrow S_{j}+1$ & $k_{i j} S_{i}$ & $S_{i}>0, i \neq j$ \\
$E_{i} \rightarrow E_{i}-1, \quad E_{j} \rightarrow E_{j}+1$ & $k_{i j} E_{i}$ & $E_{i}>0, i \neq j$ \\
$I_{i} \rightarrow I_{i}-1, \quad I_{j} \rightarrow I_{j}+1$ & $l_{i j} I_{i}$ & $I_{i}>0, i \neq j$ \\
$R_{i} \rightarrow R_{i}-1, \quad R_{j} \rightarrow R_{j}+1$ & $k_{i j} R_{i}$ & $R_{i}>0, i \neq j$ \\
Absorbing state & 0 & $\sum_{i=1}^{n} I_{i}+\sum_{i=1}^{n} E_{i}=0$ \\
\hline
\end{tabular}

\subsection{Vaccination in the random set-up}

Introduction of additional centres makes definition of optimal vaccine allocation more complicated. We calculate the health-care premium according to the equivalence principle (1.1). However, unlike (2.8), the formula for premium in stochastic model has the form

$$
\pi=\frac{1}{\mathbb{E}\left[B_{T}^{\delta}\right]}\left(c_{1} \mathbb{E}\left[A_{T}^{\delta}\right]+c_{2} \sum_{i=1}^{n} \mathbb{E}\left[\exp (-\delta T) R_{i}(T)\right]+c_{3} \nu\right)
$$


In the stochastic set-up, let $w_{i}$ be integers, such that $w_{i} \geq 0, \sum_{i=1}^{n} w_{i}=\nu$. Unlike deterministic model (2.9) with vaccine stock $\nu$, in the stochastic setup we need to clarify what is the optimal vaccine allocation. In contrast to the deterministic case, in view of small population numbers, we consider an instantaneous vaccination. Effectively, it means an immediate reduction of the number of susceptibles $S_{i}(0)$ and their transfer to the compartment $W_{i}(0)$ at the initial moment $t=0$. We apply the following procedure:

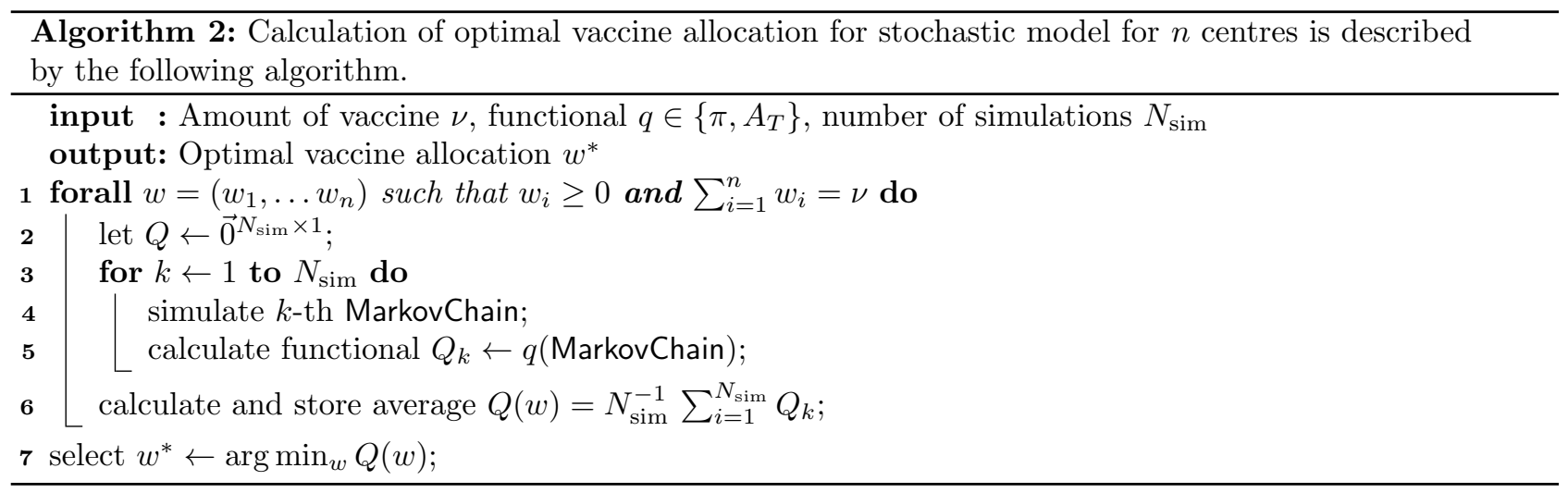

The idea of Algorithm 2 is to consider all possible vaccine allocations and simulate $N_{\text {sim }}$ Markov chains for each allocation (i.e. perform Monte Carlo method). Then we choose such allocation $w^{*}=\left(w_{1}, w_{2}, \ldots, w_{n}\right)$ that minimises the desired functional (resulting health-care premium rate $\pi$ or number of lost working days $A_{T}$ ).

After computing optimal vaccine allocation $w^{*}$, we calculate corresponding optimal premium $\pi^{*}, \pi\left(A_{T}^{*}\right)$ (i.e. use formula (3.2)), where all expected values are estimated in the same Monte Carlo simulations.

\section{NUMERICAL EXPERIMENTS}

Let us consider the deterministic model with vaccination (2.9) and the stochastic model from Section 3.3 with two centres. For numerical experiments we select the parameters which are in a reasonable agreement with the current COVID-19 epidemics in many regions worldwide: $\gamma_{i}=1 / 20,1 / 10 ; \beta_{i}=1 / 10,1 / 5 ; \sigma=1 / 5$. Thus, stands for the mean duration of the disease $T_{e}=20,10$, the mean duration of the latent period $T_{l}=5$, $\mathcal{R}_{0}=2$ and $\mathcal{R}_{0}=4$ for identical centres (for this case $\mathcal{R}_{0}=\beta / \gamma$ ). The cases $\mathcal{R}_{0}=2$ and $\mathcal{R}_{0}=4$ correspond to the mollified and unmollified scenarios of COVID-19 epidemic propagation. In the deterministic model we select $a_{1}=a_{2}=500$, i.e. the vaccination rate is 500 individuals per day. The cost of treatment for individual per day is $c_{1}=100 \$$ or $150 \$$, and $c_{2}=0$ or $100 \$$ is an additional compensation for a removed individual. Finally, $c_{3}=5 \$, 50 \$$ stands for a cost of vaccine dose. In all figures (in deterministic section) the horizontal axis corresponds to the vaccine amount required to vaccinate $V$ percents of susceptible individuals, i.e. $\nu=V S$. For example, if $S=S_{1}+S_{2}=100$, then $V=0.2$ means that the health-care office can vaccinate $20 \%$ of susceptibles, i.e. $\nu=20$ individuals. We consider $V \in[0,0.5]$ only, since it becomes extremely hard to vaccinate more than a half of population. Let the discount rate be $\delta=\log (1+r) / 365$ where $r=0.01$.

\subsection{Deterministic model}

Assume that the extinction of epidemic $T$ is the moment when the total number of infectives is smaller than $\theta=0.5 \%$ of living population, i.e. use formula (2.2). 


\subsubsection{Basic deterministic scenario. Lockdown case}

We start with the basic scenario of two identical SEIR centres. Select the initial conditions $S_{i, 0}=$ $100000, I_{i, 0}=100, E_{i, 0}=0$ and parameters $\gamma_{i}=1 / 20, i=1$, 2 . First, we consider completely isolated centres with no migration fluxes, i.e. $k_{1,2}=k_{2,1}=0$ and $l_{1,2}=l_{2,1}=0$. Our goal is to explore the vaccine allocation policies and calculate the fair insurance premium. Figure 1a, b; e, f demonstrates the results of minimizing the functional $A$ to obtain the minimal insurance premium $\pi^{*}$ in the cases $\mathcal{R}_{0}=2$ and $\mathcal{R}_{0}=4$. We conclude that $\mathcal{R}_{0}$ affects the insurance premium significantly, the premium increased threefold when the reproduction number $\mathcal{R}_{0}$ is doubled. In this case, the main parameter affecting the premium rate is $c_{1}$, the treatment cost of an individual per day. On the other hand, the cost of vaccine dose affects the premium rate negligibly, it is recommended to purchase the maximal amount of vaccine and extend the vaccination program for the maximal fraction of population to minimize the overall premium rate. The optimal vaccine allocation is not so obvious from the common sense arguments. Figure $1 \mathrm{c}$, g implies that for a small vaccine stock $V<V_{1}, V_{1} \approx 20 \%$, it should be concentrated at one centre, it does not matter which one due to the symmetry, i.e. $w_{1}=0$ or $w_{1}=1.0$. For a moderate vaccine stock in the interval $\left(V_{1}, V_{2}\right)$, it should be divided between the centres as indicated in Figure 1c, g. For a sufficiently large stock $V>V_{2}$, the vaccine should be divided equally between the centres. The vaccine allocation policy significantly affects the functional $A$, and the effect increases with the increase of the vaccine stock. The maximal reduction of the functional $A$ reaches $\sim 80 \%$ in the case $\mathcal{R}_{0}=2$ and $\sim 30 \%$ in the case $\mathcal{R}_{0}=4$.

\subsubsection{Basic deterministic scenario. Identical SEIR centres with migration}

Next, we consider two symmetric SEIR centres with migration fluxes. Let us select the initial conditions $S_{i, 0}=100000, I_{i, 0}=100, E_{i, 0}=0$ and parameters $\gamma_{i}=1 / 20, i=1,2, k_{1,2}=k_{2,1}=0.05$ and $l_{1,2}=l_{2,1}=0.005$. Comparing Figures 1 and 2 we observe that the migration fluxes do not affect the insurance premium rate. On the other hand, the optimal vaccine allocation is different: even for a small vaccine stock $V$ it should be divided between two centres. The effect of the optimal vaccine allocation is not so drastic in this case: the maximal reduction of the functional $A$ is approximately $60 \%$ for $\mathcal{R}_{0}=2$ and $\sim 30 \%$ for $\mathcal{R}_{0}=4$.

\subsubsection{Health-centre}

Assume that the epidemics has started in two centres, but only one of them has a medical facility that can cure the disease effectively. In this case, infected persons from the second centre are willing to get to the first centre to cure the disease, while infected persons from the first centre stay at home. We also assume that the second centre has a medical institution, but it is not that effective.

It is natural to assume that the first centre, which has the medical facility, is technologically more advanced and has higher population. Let the population of the first centre be $S_{1,0}=100000, E_{10}=0$ and $I_{1,0}=100$, and the population of the second centre be $S_{2,0}=10000, E_{20}=0$ and $I_{2,0}=100$. Let the recovery rates be $\gamma_{1}=1 / 10, \gamma_{2}=1 / 20$ and $\sigma=1 / 5$.

In this scenario, we assume that susceptible groups in both centres are travelling with rates $k_{12}=0.01$ and $k_{21}=0.1$, which represent the well-being of both centres. On the contrary, the infected group in the first centre almost do not travel $l_{12}=0.001$, while infectives from the second centre are coming to the health-centre with rate $l_{21}=0.1$.

The results of calculations for health-centre are shown in Figure 3. In general epidemics set-up, the results are completely different comparing to the basic scenario (Sect. 4.1.2). In the case $\beta_{i}=1 / 10$, we obtain the reproduction number $\mathcal{R}_{0}^{(1)}=1$ at the centre 1 , so the epidemic at the center will not develop. For this reason, the minimal premium rate is achieved for the purchase the vaccine stock from $10 \%$ to $20 \%$, and the premium rate grows with the cost of vaccine dose. Note that for $\beta_{i}=1 / 10$, the premium rate never exceed $1 \$$ per day. In all the cases, the optimal allocation strategy always suppresses the epidemic in the smaller centre with the smaller recovery rate $\gamma$. Note that the vaccine purchase above $25 \%$ does not reduce the value of functional $A$ significantly. This fact provides the evidence that the necessary vaccine stock does not exceed $\sim 30 \%$. In the case 


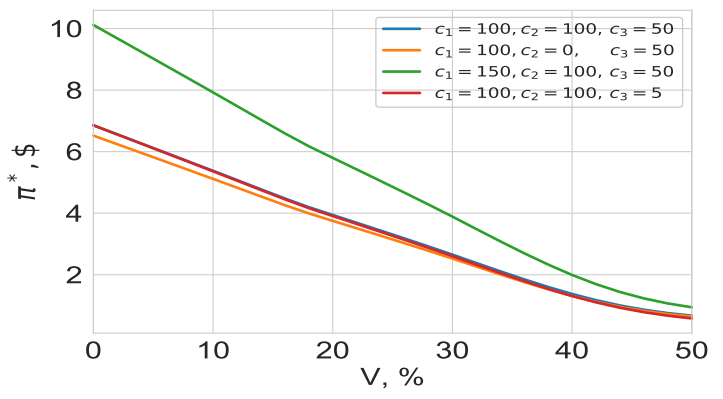

(A)



(c)

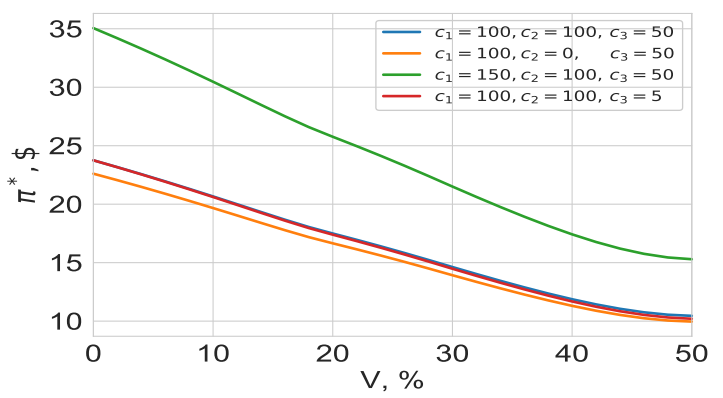

(E)



(G)



(B)

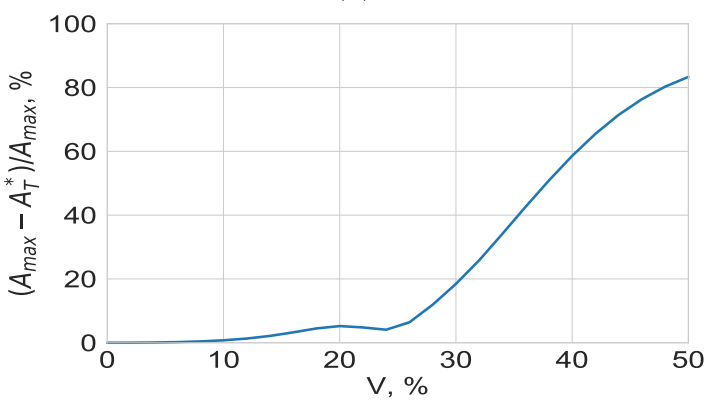

(D)

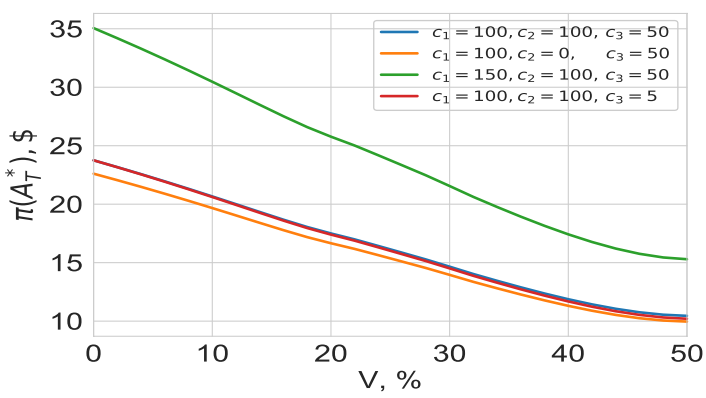

$(\mathrm{F})$



$(\mathrm{H})$

FiguRe 1. Parameters: $S_{1}(0)=100000, S_{2}(0)=100000, I_{1}(0)=100, I_{2}(0)=100, E_{1}(0)=$ $0, E_{2}(0)=0, \gamma_{1}=\gamma_{2}=\gamma=1 / 20, \sigma=1 / 5, k_{12}=k_{21}=0, l_{12}=l_{21}=0$, (a)-(d): $\beta_{1}=\beta_{2}=$ $1 / 10, \mathcal{R}_{0}=2 ;(\mathrm{e})-(\mathrm{h}): \beta_{1}=\beta_{2}=1 / 5, \mathcal{R}_{0}=4 ;(\mathrm{a}),(\mathrm{e})$ : minimal rate of insurance premium $\pi^{*}$, (b), (f): insurance premium rate in the case of minimization of functional A; (c), (g): the fraction of vaccine $w_{1}$ at centre 1 for minimization of functional $A ;(\mathrm{d}),(\mathrm{h}): \frac{A_{\max }-A_{\min }}{A_{\max }}$ - the maximal reduction of functional $A$ for optimal vaccine $V$ allocation. 




(A)

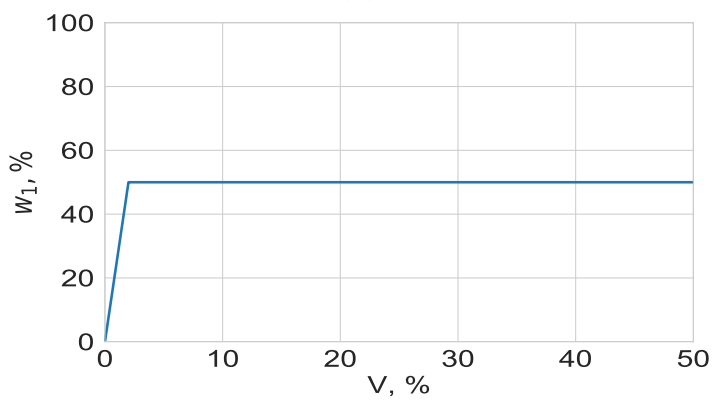

(c)

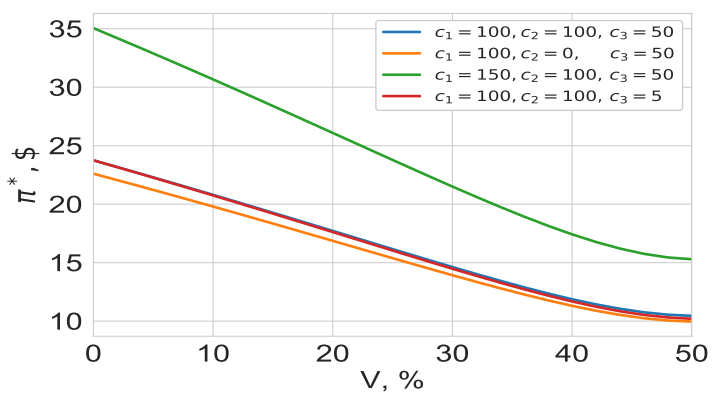

(E)

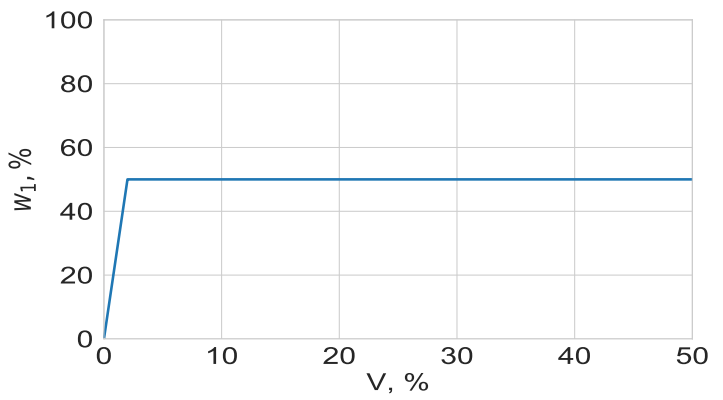

(G)

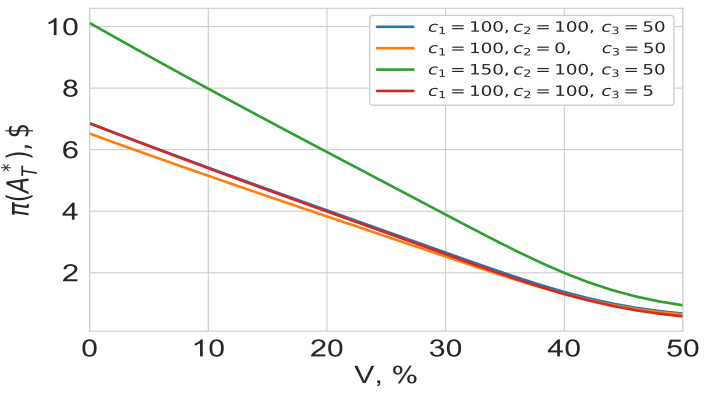

(B)



(D)

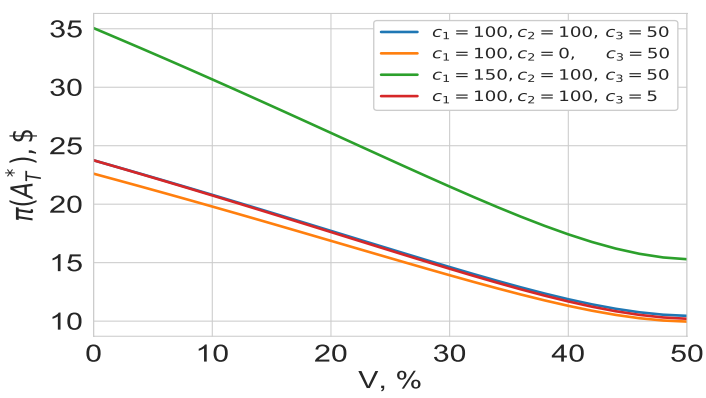

$(\mathrm{F})$

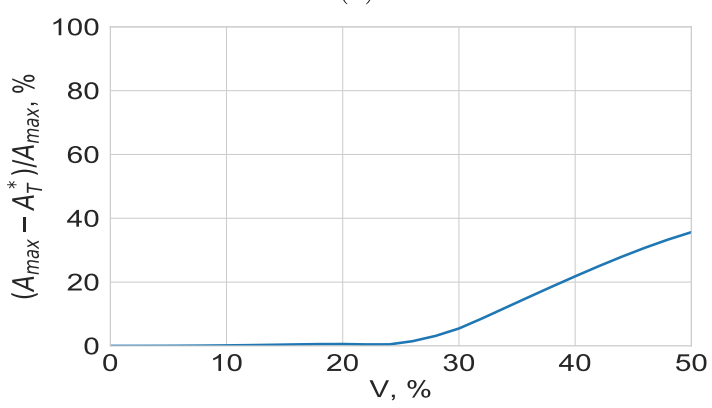

$(\mathrm{H})$

FiguRE 2. Parameters: $S_{1}(0)=100000, S_{2}(0)=100000, I_{1}(0)=100, I_{2}(0)=100, E_{1}(0)=$ $0, E_{2}(0)=0, \gamma_{1}=\gamma_{2}=\gamma=1 / 20, \sigma=1 / 5, k_{12}=k_{21}=0.05, l_{12}=l_{21}=0.005$, (a)-(d): $\beta_{1}=$ $\beta_{2}=1 / 10, \mathcal{R}_{0}=2 ;(\mathrm{e})-(\mathrm{h}): \beta_{1}=\beta_{2}=1 / 5, \mathcal{R}_{0}=4$; (a),(e): minimal rate of insurance premium $\pi^{*}$, (b), (f): minimal rate of insurance premium for minimization of functional A; (c), (g): fraction of vaccine $w_{1}$ at centre 1 for minimization of $A ;(\mathrm{d}),(\mathrm{h}): \frac{A_{\max }-A_{\min }}{A_{\max }}$ - maximal reduction of functional $A$ for optimal allocation of vaccine $V$. 


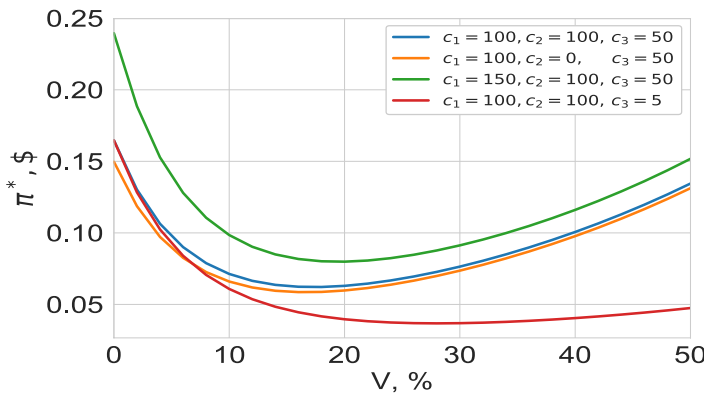

(A)

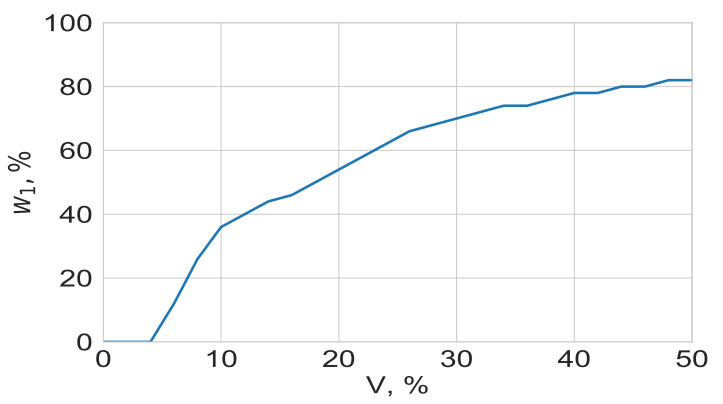

(c)

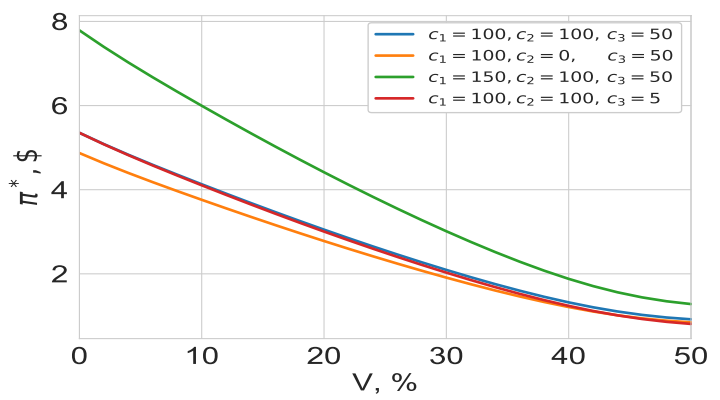

$(\mathrm{E})$

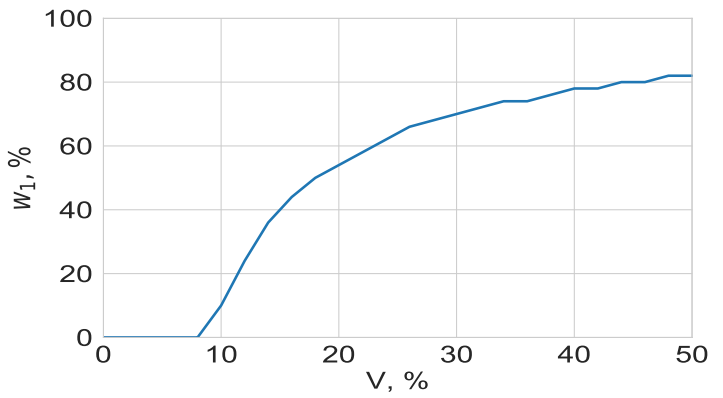

(G)

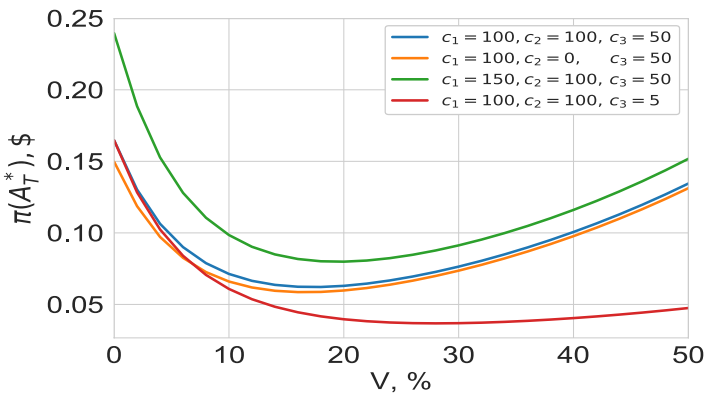

(B)



(D)

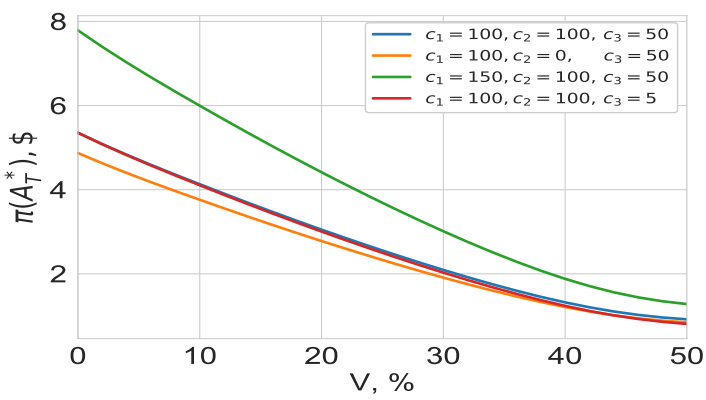

$(\mathrm{F})$

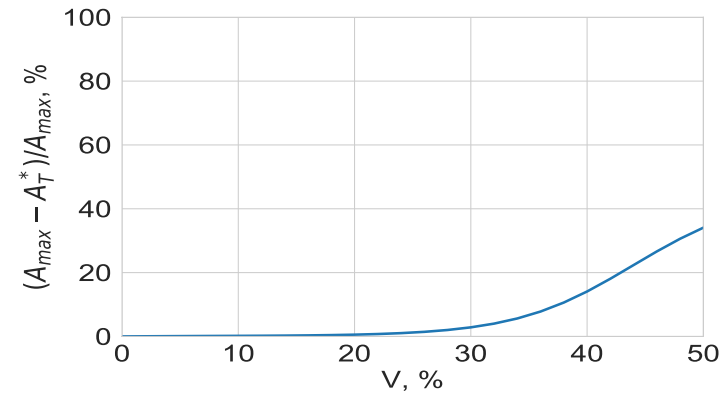

$(\mathrm{H})$

Figure 3. Parameters: $S_{1}(0)=100000, S_{2}(0)=10000, I_{1}(0)=100, I_{2}(0)=100, E_{1}(0)=$ $0, E_{2}(0)=0, \gamma_{1}=1 / 10, \gamma_{2}=1 / 20, \sigma=1 / 5, k_{12}=0.01, k_{21}=0.1, l_{12}=0.001, l_{21}=0.1$, (a)(d): $\beta_{1}=\beta_{2}=1 / 10 ;(\mathrm{e})-(\mathrm{h}): \beta_{1}=\beta_{2}=1 / 5$; (a),(e): minimal rate of insurance premium $\pi^{*}$, (b), (f): rate of insurance premium for minimization of functional A; (c), (g): fraction of vaccine $w_{1}$ at center 1 for minimization of $A ;(\mathrm{d}),(\mathrm{h}): \frac{A_{\max }-A_{\min }}{A_{\max }}$ - maximal reduction of functional $A$ for optimal vaccine allocation $V$. 
$\beta_{i}=1 / 5$, the scenario is similar to one with the symmetric centres; it is recommended to purchase the maximal amount of vaccine. The vaccine allocation is similar to the case $\beta_{i}=1 / 10$ : it is recommended to suppress the epidemic at the smaller centre first.

\subsection{Stochastic model}

In this section we abandon notation $\nu$, and let $V$ denote the available vaccine stock. Note that $V \in \mathbf{N}$ (including zero), since the stochastic model concentrates on particular individuals, so the vaccine amount is a natural number.

According to the proposed Algorithm 2 for computation of optimal vaccine allocation, for a small vaccine number $V$ we need to look for a small number of possible combinations. In fact, in the case of two centres the number $N_{V}$ of all possible allocations of vaccine amount $V$ is

$$
N_{V}=V+1
$$

For each vaccine allocation option we run $N_{\text {sim }}=10000$ Markov chains according to Table 2 .

In all figures in this section we present premiums with $95 \%$ confidence intervals

$$
\pi^{*} \pm z_{0.975} \sqrt{\frac{\operatorname{var}\left(\pi^{*}\right)}{N_{\text {sim }}}}
$$

where $z_{0.975}$ is a quantile of standard normal distribution and $\operatorname{var}\left(\pi^{*}\right)$ is calculated via Algorithm 2 as a sample variance. This form of confidence interval follows directly from Monte Carlo estimator properties.

Stochastic model is generally used to describe the infection spread throughout the population for small numbers of infected persons. The key point is that the infection can be extinct, i.e. get naturally suppressed, not causing any outbreak. For this reason, we complement Algorithm 2 with calculation of average number of extinct epidemics. For this cause, we set percentage $\theta=0.1$ of population, and say that there was no outbreak, if maximal number of infected individuals at any point of time is smaller than $10 \%$ of total susceptible population at time $t=0$ :

$$
\max _{0 \leq t \leq T} \sum_{i=1}^{2}\left[I_{i}(t)+E_{i}(t)\right]<\theta \cdot\left(S_{1,0}+S_{2,0}\right) .
$$

In general, many computationally expensive simulations should be performed to obtain precise result, which is usually done on supercomputers. Therefore, the following results are not reliable enough for practical recommendations about optimal vaccine allocation and precise premium calculations. However, they provide a satisfactory behaviour of premium dependencies.

\subsubsection{Basic stochastic scenario}

Here, we consider a basic scenario that is analogous to the deterministic case (Sect. 4.1.2). In line with [22], let initial number of susceptible individuals be $S_{i, 0}=50, E_{i, 0}=0$. However, we consider a smaller number of infected people: $I_{i, 0}=1$ for $i=1,2$. Further, let migration fluxes be $k_{12}=k_{21}=0.05$ and $l_{12}=l_{21}=0.005$. Let recovery rates be $\gamma_{i}=1 / 20$ and the infection rate be $\beta_{i}=1 / 10$ (see Fig. $4 \mathrm{a}-\mathrm{d}$ ) and $\beta_{i}=1 / 5$ (see Fig. 4e-h).

Note that the premiums $\pi^{*}$ and $\pi\left(A^{*}\right)$ are practically identical, see Figure $4 \mathrm{a}$, b and e, f. Also observe that the premium rate is a convex function, so its minimum is achieved either in the case when the population is fully vaccinated or the total cancellation of vaccination program. Next, the main parameter affecting the premium rate is $c_{1}$, i.e. the expenses for the treatment per day. During numerical simulations we assess the number of epidemics that are naturally suppressed and calculate their fractions, see Figure $4 \mathrm{~d}$. It shows that, even when we do not vaccinate the population, there is still a relatively high chance $(\sim 30 \%)$ to surpass the 


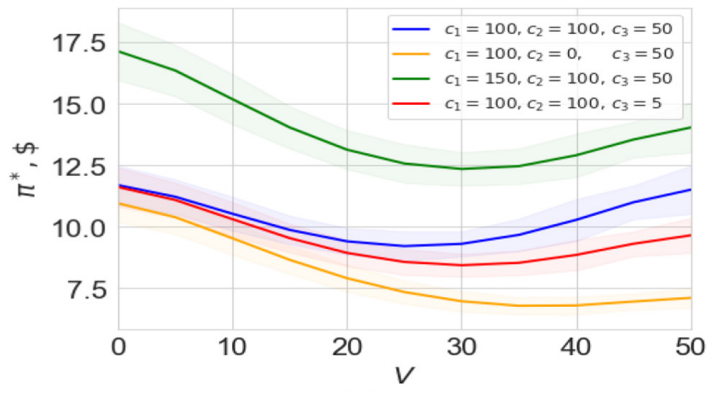

(A)



(c)

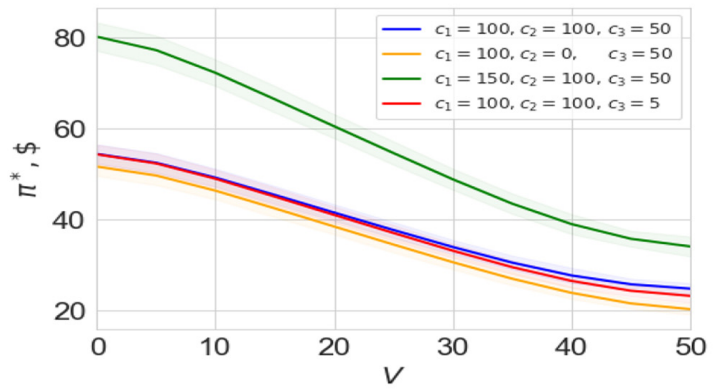

(E)

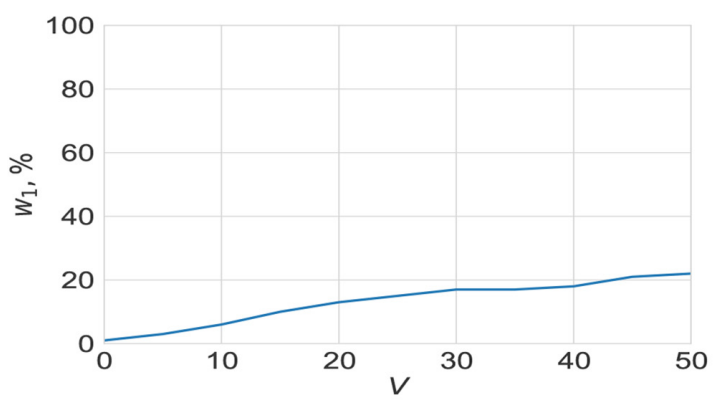

(G)



(B)

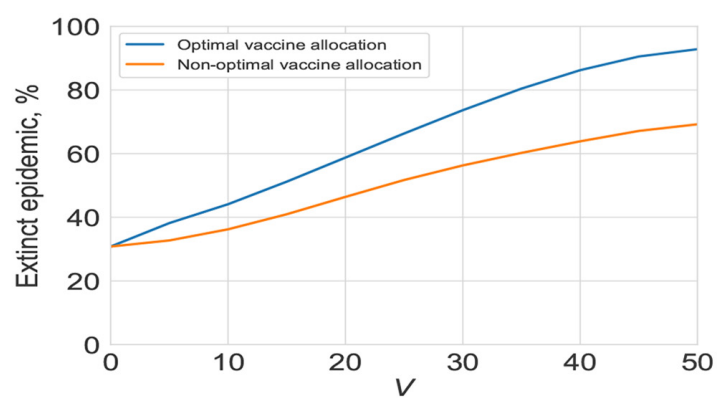

(D)

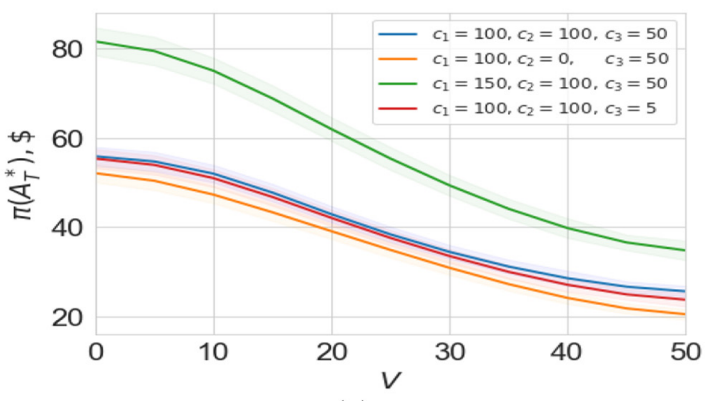

(F)



(н)

Figure 4. Parameters: $S_{1}(0)=50, S_{2}(0)=50, I_{1}(0)=1, I_{2}(0)=1, E_{1}(0)=0, E_{2}(0)=0, \gamma_{1}=$ $\gamma_{2}=\gamma=1 / 20, \sigma=1 / 5, k_{12}=k_{21}=0.05, l_{12}=l_{21}=0.005$, (a)-(d): $\beta_{1}=\beta_{2}=1 / 10, \mathcal{R}_{0}=2$; (e)-(h): $\beta_{1}=\beta_{2}=1 / 5, \mathcal{R}_{0}=4$; (a),(e): minimal rate of insurance premium $\pi^{*}$, (b), (f): insurance premium rate in the case of minimization of functional $\mathrm{A} ;(\mathrm{c}),(\mathrm{g})$ : the fraction of vaccine $w_{1}$ at centre 1 for minimization of functional $A ;(\mathrm{d}),(\mathrm{h})$ : The fractions of extinct epidemics for the optimal/best and the worst vaccine $V$ allocations. 


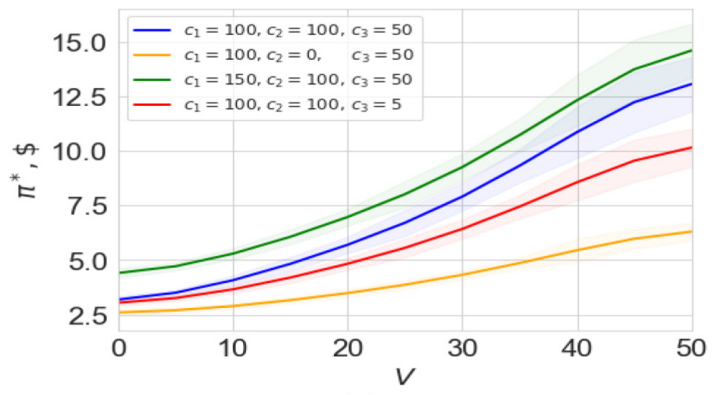

(A)

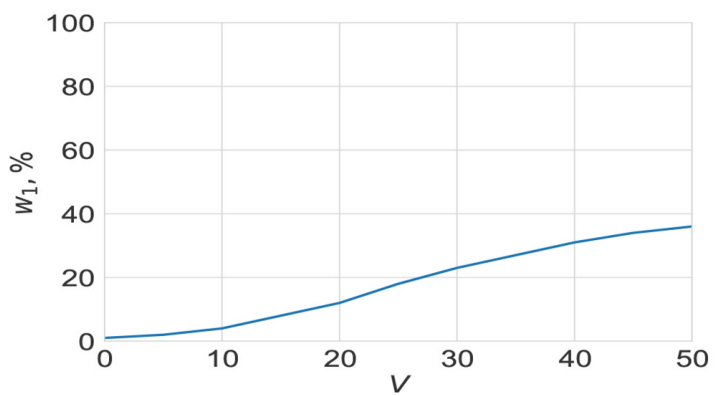

(C)

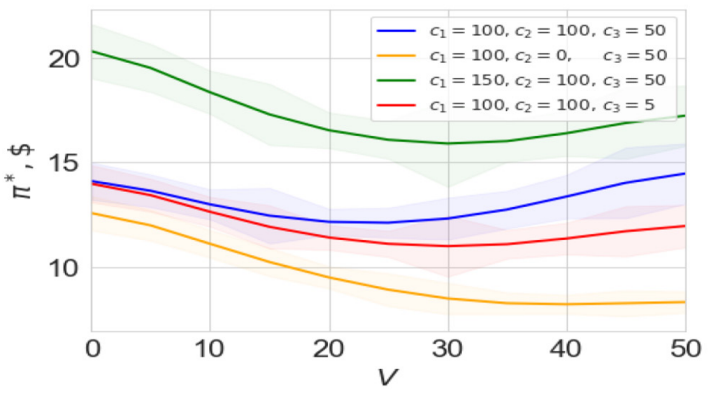

(E)

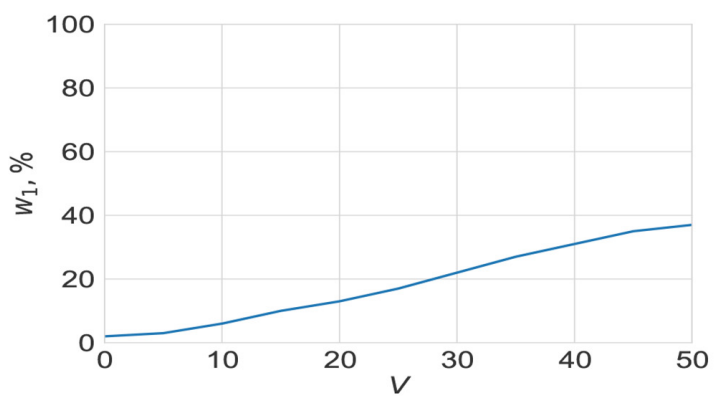

(G)

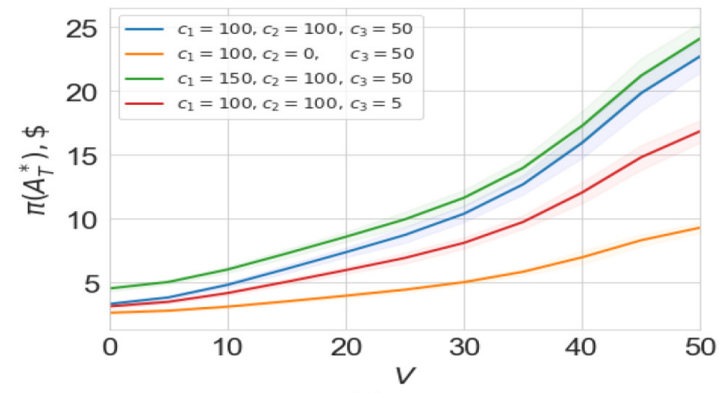

(B)



(D)

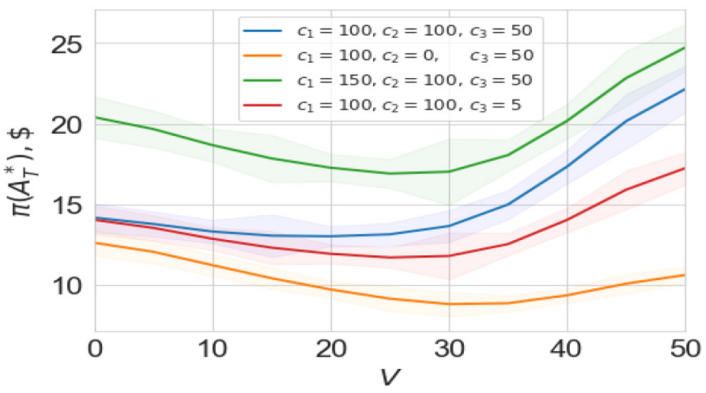

(F)

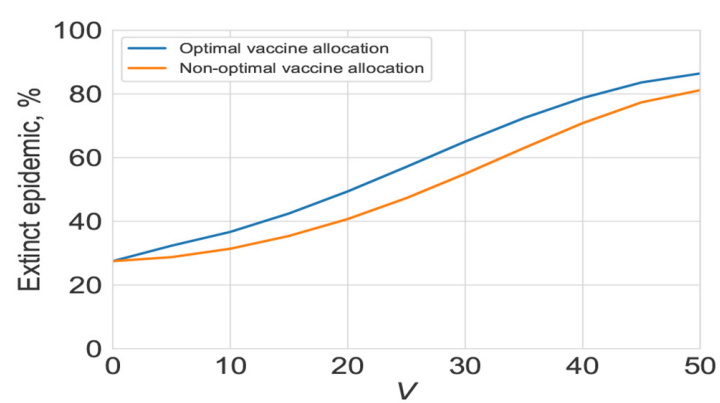

(н)

Figure 5. Parameters: $S_{1}(0)=100, S_{2}(0)=10, I_{1}(0)=1, I_{2}(0)=1, E_{1}(0)=0, E_{2}(0)=$ $0, \gamma_{1}=1 / 10, \gamma_{2}=1 / 20, \sigma=1 / 5, k_{12}=0.01, k_{21}=0.1, l_{12}=0.001, l_{21}=0.1$, (a)-(d): $\beta_{1}=\beta_{2}=$ 1/10; (e)-(h): $\beta_{1}=\beta_{2}=1 / 5$; (a),(e): minimal rate of insurance premium $\pi^{*},(\mathrm{~b})$, (f): rate of insurance premium for minimization of functional $\mathrm{A} ;(\mathrm{c}),(\mathrm{g})$ : fraction of vaccine $w_{1}$ at center 1 for minimization of $A ;(\mathrm{d}),(\mathrm{h})$ : The fractions of extinct epidemics for the optimal/best and worst vaccine $V$ allocations. 
infection. However, the optimal vaccine allocation during the vaccination program affecting a half of population may result in epidemics extinction in more then $90 \%$ of all cases. In a more severe case $\mathcal{R}_{0}=4$, observe that the premium rate $\pi$ is rather high for a relatively small population, it may be reduced by buying a considerable vaccine stock $V$ (see Fig. 4e, f). Without a vaccination program an epidemic outbreak develops in more than $90 \%$ of all cases, the vaccination of the half of population reduces its probability to $60 \%$ of all cases (see Fig. 4d).

\subsubsection{Big and small centres}

In this scenario, we consider two cities that resemble health-centre scenario considered in Section 4.1.3. Suppose that we have a big centre with an advanced treatment facility, and a small centre with a poor treatment. All infected persons try to migrate to the big centre, while infected group in the first centre generally stay at home. Susceptible group is travelling as usual.

We define an initial number of susceptibles as $S_{1,0}=100$ and $S_{2,0}=10$, and infectives as $I_{1,0}=1, I_{2,0}=1$. Migration fluxes are $k_{12}=0.1, k_{21}=0.01, l_{12}=0.1$ and $l_{21}=0.001$. Recovery rates $\gamma_{i}=1 / 20$ and the infection rates $\beta_{i}=1 / 10$ (see Fig. $5 \mathrm{a}-\mathrm{d}$ ) and $\beta_{i}=1 / 5$ (see Fig. $5 \mathrm{e}-\mathrm{h}$ ).

We observe that the minimisation of the premium rate leads to the lower values than the minimisation of the functional $A_{T}$ (see Fig. 5a, b, e, f). It happens due to the different vaccine allocation strategies. The second strategy allocates all stock of the vaccine and distributes it such that the total exposure to the disease (functional $\left.A_{T}\right)$ is minimised. To the contrary, the first strategy only aims to minimise the resulting premium. Therefore, it searches for the balance between preventing the epidemic (minimisation of $A_{T}$ ) and keeping people nonvaccinated (maximisation of $B_{T}$ ), see formula (3.2). That way, more people are kept susceptible to the disease ${ }^{1}$ and they are forced to buy the health insurance policy, which allows to reduce the premium rate.

In both optimality approaches the best strategy is to vaccinate the small centre first. When the stock is higher than 10 (the population of the second centre) the "minimisation of $A_{T}$ " strategy allocates the surplus to the first centre, whereas the "premium minimisation" throws the surplus away (i.e. the surplus is not used). That way the disease has a chance to develop in the big centre, prolonging the duration of the epidemic. As a result, the higher income for the health-care office is generated and the premium rate is reduced.

Also we observe an unusual behaviour in the case $\beta_{i}=1 / 10$ - the premium rate grows with the extension of the vaccination program (see Fig. $5 \mathrm{a}$, b). The reason is that nearly $90 \%$ of all epidemics extinct even without any vaccination (see Fig. $5 \mathrm{~d}$ ). So, the bulk of the premium is formed by the cost of the vaccine stock. In the case $\beta_{i}=1 / 5$, the minimal insurance rate is achieved for vaccine stock from $20 \%$ to $40 \%$ (see Fig. $5 \mathrm{e}$ ). If the vaccine is not expensive, say $c_{3}=5 \$$, the best policy is to purchase the maximal stock. With the vaccination program on the level $V=50 \%$, the probability of epidemic extinction approaches $\sim 90 \%$.

\section{Conclusion}

Modern epidemiology is a consistent theory that provides a variety of models, and the tool-kit to study the dependencies of the solutions from their parameters and the initial conditions. In general, all models are supposed to study individuals with certain probabilities of passing/acquiring the infection, migration, immunisation, etc. However, the theory shows that the solution offered by a stochastic model, with a lot of time steps, is approaching the solution to a dynamic problem with initial conditions in terms of fractions of initially infected population. At the same time, when the number of infected individuals is small, the epidemic has a high probability of being naturally suppressed, which is only possible in a stochastic model.

In this work, we concentrate on the simplest SEIR model with two centres and migration fluxes. We study both dynamic and stochastic models in different "what-if" scenarios, and look for the optimal vaccine allocation between the centres. The expenses for treatment of individuals who are not vaccinated are covered by a mandatory health-care policy. Taking into account the dynamics of the epidemic, we evaluate the health-care premium rate, paid from a public fund or privately, based on the equivalence principle.

\footnotetext{
${ }^{1}$ Our approach allows to consider cases when a portion of the vaccine is not allocated to the centres, i.e. some vaccine is bought, but not sold.
} 
We propose two measures of "optimality" - one being financially driven, while the second aims to maintain the population healthy. Generally, both approaches result in very similar premium rates. If both measures lead to different results, it would be reasonable to consider their linear combination with proper coefficients.

The results of numerical integration in Section 4.1 and stochastic simulations in Section 4.2 show some interesting features of vaccine allocation strategies and the resulting premiums such as the switch from the policy of concentration vaccine at weak spots to more uniform distribution with the increasing stock. The calculations are made for infections features with different hostility levels. The main concern here is to investigate the allocation strategies under a low vaccine stock.

Future works may be based on more detailed and advanced models, such as

- a fatal epidemic (when the removed group is replaced by two separate compartments of recovered/succumbed individuals);

- extension to SEIRS model (recovered individuals lose immunity after some time and become susceptible again); and to SLIAR and Erlang SEIR models;

- different vaccination policies for different age groups;

- individuals who did not get the vaccine from the health-care office receive a right to purchase it at a certain price.

Finally, the selection of the models' parameters should be specified by experts, providing more accurate and applicable results.

\section{Appendix A. Calculation of Reproduction number $\mathcal{R}_{0}$}

Finally, we compute the reproduction number $\mathcal{R}_{0}$ for the system (2.1) by the next generation matrix method, see $[11,26,35]$. For this aim, write $(2.1)$ in the form

$$
\dot{x}=f(x)
$$

where $x=\left[x_{1}, x_{2}, x_{3}, x_{4}, x_{5}, x_{6}, x_{7}, x_{8}\right]^{T}=\left[S_{1}, E_{1}, I_{1}, R_{1}, S_{2}, E_{2}, I_{2}, R_{2}\right]^{T}$.

As usual, the fix points $x_{e q}$ of the system are defined by $f\left(x_{e q}\right)=0$. In the case when the exposed and infected individuals are absent at the state $x_{e q}$, this is a disease free equilibrium (DFE). The stability analysis of DFE in terms of the reproduction number $\mathcal{R}_{0}$ is presented in $[35,36]$. In particular, it is proven that for $\mathcal{R}_{0}>1 \mathrm{DFE}$ is unstable, and even a single infected individual would trigger an outbreak. On the other hand, for $\mathcal{R}_{0}<1 \mathrm{DFE}$ is locally asymptotically stable.

It could be easily checked that $x_{0}=\left(S_{1}(0), S_{2}(0), 0,0,0,0,0,0\right)$ is DFE for the system (A.1). Let us separate $x_{I}=\left(E_{1}, E_{2}, I_{1}, I_{2}\right)$, i.e. the infectious components of the vector $x$. Thus,

$$
\dot{x}_{I}=f_{I}(x) .
$$

$f_{I}(x)=\mathcal{F}_{I}-\mathcal{V}_{I}$ where $\mathcal{F}_{I}$ stands for a vector-column of the new infectious input, and $\mathcal{V}_{I}$ is a vector-column of infectious transfer between compartments:

$$
\mathcal{F}_{I}=\left[\begin{array}{c}
\frac{\beta_{1} S_{1} I_{1}}{N_{1}} \\
\frac{\beta_{2} S_{2} I_{2}}{N_{2}} \\
0 \\
0
\end{array}\right], \mathcal{V}_{I}=\left[\begin{array}{c}
\sigma E_{1}+k_{12} E_{1}-k_{21} E_{2} \\
\sigma E_{2}+k_{21} E_{2}-k_{12} E_{1} \\
-\sigma E_{1}+\gamma I_{1}+l_{12} I_{1}-l_{21} I_{2} \\
-\sigma E_{2}+\gamma I_{2}+l_{21} I_{2}-l_{12} I_{1}
\end{array}\right]
$$

Next, compute the matrix $F=\frac{\partial \mathcal{F}_{I}}{\partial x}\left(x_{0}\right)$, with non-negative elements, and the non-singular matrix $V=$ $\frac{\partial \mathcal{V}_{I}}{\partial x}\left(x_{0}\right)$, the so-called $M$-matrix, see [5]. More precisely, $M$-matrix has the property that off-diagonal elements 
are non-positive and all elements of its inverse matrix are non-negative. Then

$$
\begin{aligned}
& F=\left[\begin{array}{cccc}
0 & 0 & \frac{\beta_{1} S_{1}(0)}{N_{1}} & 0 \\
0 & 0 & 0 & \frac{\beta_{2} S_{2}(0)}{N_{2}} \\
0 & 0 & 0 & 0 \\
0 & 0 & 0 & 0
\end{array}\right] \\
& V=\left[\begin{array}{cccc}
\sigma+k_{12} & -k_{21} & 0 & 0 \\
-k_{12} & \sigma+k_{21} & 0 & 0 \\
-\sigma & 0 & \gamma_{1}+l_{12} & -l_{21} \\
0 & -\sigma & -l_{12} & \gamma_{2}+l_{21}
\end{array}\right] \\
& V^{-1}=\left[\begin{array}{cccc}
\frac{\sigma+k_{21}}{\sigma A} & \frac{k_{21}}{\sigma A} & 0 & 0 \\
\frac{k_{12}}{\sigma A} & \frac{\sigma+k_{12}}{\sigma A} & 0 & 0 \\
\frac{\sigma\left(\gamma_{2}+l_{21}\right)+\gamma_{2} k_{21}+l_{21}\left(k_{12}+k_{21}\right)}{A B} & \frac{l_{21} A+\gamma_{2} k_{21}}{A B} & \frac{\gamma_{2}+l_{21}}{B} & \frac{l_{21}}{B} \\
\frac{l_{12} A+\gamma_{1} k_{12}}{A B} & \frac{\sigma\left(\gamma_{1}+l_{12}\right)+\gamma_{12}+l_{12}\left(k_{12}+k_{21}\right)}{A B} & \frac{l_{12}}{B} & \frac{\gamma_{1}+l_{12}}{B}
\end{array}\right]
\end{aligned}
$$

where $A=\left(\sigma+k_{12}+k_{21}\right), B=\left(\gamma_{1} \gamma_{2}+\gamma_{1} l_{21}+\gamma_{2} l_{12}\right)$. Finally, compute

$F V^{-1}=\left[\begin{array}{cccc}\frac{\beta_{1} S_{1}(0)\left[\sigma\left(\gamma_{2}+l_{21}\right)+\gamma_{2} k_{21}+l_{21}\left(k_{12}+k_{21}\right)\right]}{N_{1} A B} & \frac{\beta_{1} S_{1}(0)\left(l_{21} A+\gamma_{2} k_{21}\right)}{N_{1} A B} & \frac{\beta_{1} S_{1}(0)\left(\gamma_{2}+l_{21}\right)}{N_{1} B} & \frac{\beta_{1} S_{1}(0) l_{21}}{N_{1} B} \\ \frac{\beta_{2} S_{2}(0)\left[l_{12} A+\gamma_{1} k_{12}\right]}{N_{2} A B} & \frac{\beta_{2} S_{2}(0)\left[\sigma\left(\gamma_{1}+l_{12}\right)+\gamma_{1} k_{12}+l_{12}\left(k_{12}+k_{21}\right)\right]}{N_{2} A B} & \frac{\beta_{2} S_{2}(0) l_{12}}{N_{2} B} & \frac{\beta_{2} S_{2}(0)\left[\gamma_{1}+l_{12}\right]}{N_{2} B} \\ 0 & 0 & 0 & 0 \\ 0 & 0 & 0 & 0\end{array}\right]$

In view of the fact that rows 3 and 4 are zeros, non-zero eigenvalues of the matrix (A.7) coincide with that of the matrix (A.8)

$$
F V^{-1}=\left[\begin{array}{cc}
\frac{\beta_{1} S_{1}(0)\left[\sigma\left(\gamma_{2}+l_{21}\right)+\gamma_{2} k_{21}+l_{21}\left(k_{12}+k_{21}\right)\right]}{N_{1} A B} & \frac{\beta_{1} S_{1}(0)\left(l_{21} A+\gamma_{2} k_{21}\right)}{N_{1} A B} \\
\frac{\beta_{2} S_{2}(0)\left[l_{12} A+\gamma_{1} k_{12}\right]}{N_{2} A B} & \frac{\beta_{2} S_{2}(0)\left[\sigma\left(\gamma_{1}+l_{12}\right)+\gamma_{1} k_{12}+l_{12}\left(k_{12}+k_{21}\right)\right]}{N_{2} A B}
\end{array}\right]
$$

Note that $F V^{-1}$ is called the next generation matrix, and the reproduction number $\mathcal{R}_{0}$ is computed as follows:

$$
\mathcal{R}_{0}=\rho\left(F V^{-1}\right)
$$

where $\rho$ stands for the spectral radius. In the general case,

$$
\mathcal{R}_{0}=\frac{1}{2}\left(a+d+\sqrt{a^{2}+4 b c-2 a d+d^{2}}\right),
$$


where

$$
\begin{aligned}
& a=\frac{\beta_{1} S_{1}(0)\left[\sigma\left(\gamma_{2}+l_{21}\right)+\gamma_{2} k_{21}+l_{21}\left(k_{12}+k_{21}\right)\right]}{N_{1}\left(\sigma+k_{12}+k_{21}\right)\left(\gamma_{1} \gamma_{2}+\gamma_{1} l_{21}+\gamma_{2} l_{12}\right)}, \\
& b=\frac{\beta_{1} S_{1}(0)\left(l_{21}\left(\sigma+k_{12}+k_{21}\right)+\gamma_{2} k_{21}\right)}{N_{1}\left(\sigma+k_{12}+k_{21}\right)\left(\gamma_{1} \gamma_{2}+\gamma_{1} l_{21}+\gamma_{2} l_{12}\right)}, \\
& c=\frac{\beta_{2} S_{2}(0)\left[l_{12}\left(\sigma+k_{12}+k_{21}\right)+\gamma_{1} k_{12}\right]}{N_{2}\left(\sigma+k_{12}+k_{21}\right)\left(\gamma_{1} \gamma_{2}+\gamma_{1} l_{21}+\gamma_{2} l_{12}\right)}, \\
& d=\frac{\beta_{2} S_{2}(0)\left[\sigma\left(\gamma_{1}+l_{12}\right)+\gamma_{1} k_{12}+l_{12}\left(k_{12}+k_{21}\right)\right]}{N_{2}\left(\sigma+k_{12}+k_{21}\right)\left(\gamma_{1} \gamma_{2}+\gamma_{1} l_{21}+\gamma_{2} l_{12}\right)}
\end{aligned}
$$

Let us consider two simple particular cases:

1. Two independent SEIR centres without migration: $k_{12}=k_{21}=l_{12}=l_{21}=0$. In this case $b=c=0, a=$ $\frac{\beta_{1} S_{1}(0)}{\gamma_{1} N_{1}}, d=\frac{\beta_{2} S_{2}(0)}{\gamma_{2} N_{2}}$.

$$
\left.\mathcal{R}_{0}=\frac{1}{2}(a+d+|a-d|)\right)=\max (a, d)=\max \left(\frac{\beta_{1} S_{1}(0)}{\gamma_{1} N_{1}}, \frac{\beta_{2} S_{2}(0)}{\gamma_{2} N_{2}}\right)
$$

i.e. $\mathcal{R}_{0}$ coincides with the maximal reproduction number for two independent centres. The epidemic is suppressed iff both reproduction numbers are less than 1 , i.e. $\frac{\beta_{1} S_{1}(0)}{\gamma_{1} N_{1}}<1$ and $\frac{\beta_{2} S_{2}(0)}{\gamma_{2} N_{2}}<1$.

2. Two identical centres with symmetrical fluxes: $S_{1}(0)=S_{2}(0)=S(0), N_{1}=N_{2}=N, \gamma_{1}=\gamma_{2}=\gamma, \beta_{1}=$ $\beta_{2}=\beta, k_{12}=k_{21}=k, l_{12}=l_{21}=l$. In this case $a=d=\frac{\beta S(0)[\sigma(\gamma+l)+\gamma k+2 k l]}{N(\sigma+2 k) \gamma(\gamma+2 l)}, b=c=\frac{\beta S(0)(l(\sigma+2 k)+\gamma k)}{N(\sigma+2 k) \gamma(\gamma+2 l)}$.

$$
\mathcal{R}_{0}=a+b=\frac{\beta S(0)(\sigma \gamma+2 \sigma l+2 \gamma k+4 k l)}{N(\sigma+2 k) \gamma(\gamma+2 l)}=\frac{\beta S(0)(\sigma+2 k)(\gamma+2 l)}{N(\sigma+2 k) \gamma(\gamma+2 l)}=\frac{\beta S(0)}{\gamma N}
$$

Summing up, in the simplest cases the reproduction number $\mathcal{R}_{0}$ for the system $(2.1)$ coincides with that for the classical SIR and SEIR models with the single centre.

\section{REFERENCES}

[1] C.L. Althaus, Estimating the reproduction number of Ebola virus (EBOV) during the 2014 outbreak in West Africa. PLOS Curr. Outbreaks (2014).

[2] O.M. Araz, A. Galvani and L.A. Meyers, Geographic prioritization of distributing pandemic influenza vaccines. Health Care Manag. Sci. 15 (2012) 175-187.

[3] N.T. Bailey, Mathematical Theory of Epidemics, Charles Griffin (1957).

[4] E. Bakare, A. Nwagwo and E. Danso-addo, Optimal control analysis of an SIR epidemic model with constant recruitment. Int. J. Appl. Math. Res. 3 (2014).

[5] A. Berman and R.J. Plemmons, Nonnegative Matrices in the Mathematical Sciences. Society for Industrial and Applied Mathematics (1994).

[6] D. Bernoulli, Essai d'une nouvelle analyse de la mortalité causée par la petite vérole et des avantages de l'inoculation pour la prévenir. Mémoires de mathématique et de physique, presentés à l'Académie royale des sciences, par divers sçavans $\mathcal{E}^{3}$ lûs dans ses assemblées (1760) 1-45.

[7] B. Bolker and B.T. Grenfel, Space, persistence and dynamics of measles epidemics. Philos. Trans. Royal Soc. London B 348 (1995) 309-320.

[8] J. Burton, L. Billings, D.A. Cummings and I.B. Schwartz, Disease persistence in epidemiological models: the interplay between vaccination and migration. Math. Biosci. 239 (2012) 91-96.

[9] A.A. Chernov, M.Y. Kelbert and A.A. Shemendyuk, Optimal vaccine allocation during the mumps outbreak in two SIR centres. Math. Med. Biol. 37 (2019) 303-312.

[10] D.J. Daley and J. Gani, Epidemic Modelling: An Introduction. Cambridge Studies in Mathematical Biology, Cambridge University Press (1999).

[11] O. Diekmann, J.A.P. Heesterbeek and J.A.J. Metz, On the definition and the computation of the basic reproduction ratio $r_{0}$ in models for infectious diseases in heterogeneous populations. J. Math. Biol. 28 (1990) 365-382. 
[12] L.E. Duijzer, W.L. van Jaarsveld, J. Wallinga and R. Dekker, Dose-optimal vaccine allocation over multiple populations. Prod. Oper. Manag. 27 (2018) 143-159.

[13] R. Feng and J. Garrido, Actuarial applications of epidemiological models. North Am. Actuarial J. 15 (2011).

[14] N. Ferguson, D. Laydon, G. Nedjati Gilani, N. Imai, K. Ainslie, M. Baguelin, S. Bhatia, A. Boonyasiri, Z. Cucunuba Perez, G. Cuomo-Dannenburg, A. Dighe, I. Dorigatti, H. Fu, K. Gaythorpe, W. Green, A. Hamlet, W. Hinsley, L. Okell, S. Van Elsland, H. Thompson, R. Verity, E. Volz, H. Wang, Y. Wang, P. Walker, P. Winskill, C. Whittaker, C. Donnelly, S. Riley and A. Ghani, Impact of non-pharmaceutical interventions (NPIs) to reduce COVID-19 mortality and healthcare demand, report, Imperial College London, March 2020.

[15] P.E.M. Fine, Herd immunity: history, theory, practice. Epidemiolog. Rev. 15 (1993) 265-302.

[16] W. Gleißner, The spread of epidemics. Appl. Math. Comput. 27 (1988) 167-171.

[17] E. Goldstein, A. Apolloni, B. Lewis, J.C. Miller, M. Macauley, S. Eubank, M. Lipsitch and J. Wallinga, Distribution of vaccine/antivirals and the 'least spread line' in a stratified population. J. Roy. Soc. Interface 7 (2010) 755-764.

[18] H.W. Hethcote, An immunization model for a heterogeneous population. Theor. Popul. Biol. 14 (1978) $338-349$.

[19] W.O. Kermack and A.G. McKendrick, A contribution to the mathematical theory of epidemics. Proc. Roy. Soc. A 115 (1927) $700-721$.

[20] M. Kretzschmar, P.F.M. Teunis and R.G. Pebody, Incidence and reproduction numbers of pertussis: estimates from serological and social contact data in five European countries. PLOS Med. 7 (2010) 1-10.

[21] S. Lee, M. Golinski and G. Chowell, Modeling optimal age-specific vaccination strategies against pandemic influenza. Bull. Math. Biol. 74 (2012) 958-980.

[22] C. Lefèvre, P. Picard and M. Simon, Epidemic risk and insurance coverage. J. Appl. Probab. 54 (2017) $286-303$.

[23] Y. Liu, A.A. Gayle, A. Wilder-Smith and J. Rocklöv, The reproductive number of COVID-19 is higher compared to SARS coronavirus. J. Travel Med. 27 (2020) taaa021.

[24] I.M. Longini, E. Ackerman and L.R. Elveback, An optimization model for influenza a epidemics. Math. Biosci. 38 (1978) 141-157.

[25] G. Macdonald, The epidemiology and control of malaria. Oxford University Press, London (1957).

[26] M. Martcheva, An Introduction to Mathematical Epidemiology. Springer US (2015).

[27] L. Matrajt, M.E. Halloran and I.M. Longini, Jr, Optimal vaccine allocation for the early mitigation of pandemic influenza. PLOS Comput. Biol. 9 (2013) 1-15.

[28] L. Matrajt and I.M. Longini, Jr, Optimizing vaccine allocation at different points in time during an epidemic. PLOS ONE 5 (2010) $1-11$.

[29] S.D. Mylius, T.J. Hagenaars, A.K. Lugnér and J. Wallinga, Optimal allocation of pandemic influenza vaccine depends on age, risk and timing. Vaccine 26 (2008) 3742-3749.

[30] A. Reich, Properties of premium calculation principles. Insurance 5 (1986) 97-101.

[31] J. Riou and C.L. Althaus, Pattern of early human-to-human transmission of Wuhan 2019 novel coronavirus (2019-nCoV), December 2019 to January 2020. Eurosurveillance 25 (2020).

[32] R. Ross, The Prevention of Malaria, John Murray Publishing House, London (1910).

[33] I. Sazonov, M. Kelbert and M.B. Gravenor, A two-stage model for the SIR outbreak: Accounting for the discrete and stochastic nature of the epidemic at the initial contamination stage. Math. Biosci. 234 (2011) 108-117.

[34] I. Sazonov, M. Kelbert and M.B. Gravenor, A new view on migration processes between SIR centra: an account of the different dynamics of host and guest. J. Infect. Non Infect. Dis. 1 (2015).

[35] P. van den Driessche, Reproduction numbers of infectious disease models. Infect. Disease Model. 2 (2017) $288-303$.

[36] P. van den Driessche and J. Watmough, Reproduction numbers and sub-threshold endemic equilibria for compartmental models of disease transmission. Math. Biosci. 180 (2002) 29-48.

[37] E.C. Yuan, D.L. Alderson, S. Stromberg and J.M. Carlson, Optimal vaccination in a stochastic epidemic model of two non-interacting populations. PLOS ONE 10 (2015) 1-25. 